ARTICLE

https://doi.org/10.1038/s41467-020-14516-5

\title{
Structural insights into selective interaction between type lla receptor protein tyrosine phosphatases and Liprin- $\alpha$
}

Maiko Wakita1,2,3,9, Atsushi Yamagata1,2,3,4,7,9, Tomoko Shiroshima1,2,4, Hironori Izumi ${ }^{5}$, Asami Maeda,2,4, Mizuki Sendo ${ }^{5}$, Ayako Imai ${ }^{5}$, Keiko Kubota2 ${ }^{2}$ Sakurako Goto-Ito ${ }^{1,2,4}$, Yusuke Sato 1,2,3,4,8, Hisashi Mori (0 ${ }^{5}$, Tomoyuki Yoshida ${ }^{5,6 \star}$ \& Shuya Fukai (1) 1,2,3,4*

Synapse formation is induced by transsynaptic interaction of neuronal cell-adhesion molecules termed synaptic organizers. Type lla receptor protein tyrosine phosphatases (Ila RPTPs) function as presynaptic organizers. The cytoplasmic domain of Ila RPTPs consists of two phosphatase domains, and the membrane-distal one (D2) is essential for synapse formation. Liprin- $\alpha$, which is an active zone protein critical for synapse formation, interacts with D2 via its C-terminal domain composed of three tandem sterile alpha motifs (tSAM). Structural mechanisms of this critical interaction for synapse formation remain elusive. Here, we report the crystal structure of the complex between mouse PTP D2 and Liprin- $\alpha 3$ tSAM at $1.91 \AA$ resolution. PTPS D2 interacts with the $N$-terminal helix and the first and second SAMs (SAM1 and SAM2, respectively) of Liprin- $\alpha 3$. Structure-based mutational analyses in vitro and in cellulo demonstrate that the interactions with Liprin- $\alpha$ SAM1 and SAM2 are essential for the binding and synaptogenic activity.

\footnotetext{
${ }^{1}$ Institute for Quantitative Biosciences, The University of Tokyo, Tokyo 113-0032, Japan. ${ }^{2}$ Synchrotron Radiation Research Organization, The University of Tokyo, Tokyo 113-0032, Japan. ${ }^{3}$ Department of Computational Biology and Medical Sciences, Graduate School of Frontier Sciences, The University of Tokyo, Chiba 277-8561, Japan. ${ }^{4}$ CREST, JST, Saitama 332-0012, Japan. ${ }^{5}$ Department of Molecular Neuroscience, Graduate School of Medicine and Pharmaceutical

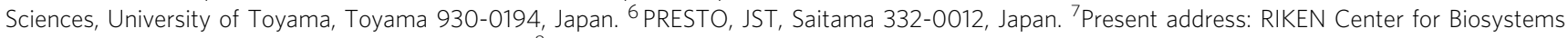
Dynamics Research, Kanagawa 230-0045, Japan. ${ }^{8}$ Present address: Center for Research on Green Sustainable Chemistry, Tottori University, Tottori 680-8582, Japan. ${ }^{9}$ These authors contributed equally: Maiko Wakita, Atsushi Yamagata *email: toyoshid@med.u-toyama.ac.jp; fukai@iam.u-tokyo.ac.jp
} 
S ynaptic organizers are a class of neuronal adhesion or secretory proteins capable of inducing synaptic differentiation, and play important roles in formation and maturation of neuronal synapses ${ }^{1,2}$. Type IIa receptor protein tyrosine phosphatases (IIa RPTPs) function as presynaptic organizers. Dysfunctions of IIa RPTPs and their postsynaptic partners are associated with neurodevelopmental disorders, such as autism spectrum disorders (ASD), intellectual disability, or schizophrenia ${ }^{1,3,4}$. The vertebrate IIa RPTP family consists of three members, PTP $\delta$, PTP $\sigma$, and LAR, which share the same domain architecture with a large $\mathrm{N}$-terminal extracellular domain, a single transmembrane segment, and a cytoplasmic domain (Fig. 1a) ${ }^{3,4}$. The N-terminal extracellular domain comprises three immunoglobulin-like (Ig) domains and four or eight fibronectin type III (FN) domains ${ }^{5}$. The extracellular domain mediates a heterophilic, transsynaptic interaction with various postsynaptic organizers such as Netrin-G ligand 3 (NGL-3) ${ }^{6,7}$, Tropomyosin kinase $\mathrm{C}(\mathrm{TrkC})^{8}$, Interleukin-1 receptor accessory protein-like 1 (IL1RAPL1) ${ }^{9}$, Interleukin-1 receptor accessory protein (IL-1RAcP) ${ }^{10}$, Slit- and Trk-like family protein (Slitrk) 1 -Slitrk6 $6^{11,12}$, synaptic adhesion-like molecule (SALM) $3^{13}$, and SALM5 ${ }^{14}$. All of these postsynaptic organizers except NGL-3 interact with the Ig domains of IIa RPTPs ${ }^{15-20}$. Their selective interactions are controlled by two mini-exon-derived peptides (meA and meB peptides), which are located within the Ig2 domain and in the junction between the Ig2 and Ig3 domains, respectively.

The cytoplasmic domain of IIa RPTPs comprises two tandem phosphatase domains ${ }^{3,4}$. The membrane-proximal phosphatase domain (D1) is catalytically active, whereas the membrane-distal one (D2) is inactive. The D2 domain of IIa RPTPs directly interacts with several synaptic proteins including Liprin- $\alpha^{21,22}$, Caskin $^{23}$, and $\mathrm{Trio}^{24}$. In heterologous synaptogenic co-culture assays using short hairpin RNA (shRNA)-mediated PTPo knockdown neurons and HEK293T cells expressing TrkC or Slitrk1, re-expression of phosphatase-dead PTP $\sigma$ mutants could partially recover the synaptogenic activity in PTP $\sigma$-knockdown neurons but that of a D2-lacking mutant could not ${ }^{25}$. The intracellular interactions of PTPб with synaptic proteins via the D2 domain likely play more critical roles in synaptogenesis than the phosphatase activity catalyzed by the D1 domain.

Liprin- $\alpha$ is an active zone protein and the first protein identified as an intracellular binding partner of IIa RPTPs ${ }^{21}$. Liprin- $\alpha$ belongs to the Liprin family, which is highly conserved from invertebrates to mammals 22,26 . The vertebrate Liprin family is classified into three groups: Liprin- $\alpha$, Liprin- $\beta$, and KazrinE ${ }^{22,27}$. Mammals possess four Liprin- $\alpha$ isoforms (Liprin- $\alpha 1-$ Liprin- $\alpha 4$ ), two Liprin- $\beta$ isoforms (Liprin- $\beta 1$ and $-\beta 2$ ), and KazrinE. On the other hand, invertebrates possess a single set of Liprin- $\alpha$, $-\beta$, and $-\gamma^{22,28}$. Liprin- $\gamma$ is the closest homolog of KazrinE. Among them, Liprin- $\alpha$ and $-\gamma$ can reportedly bind to the D2 domain of IIa RPTPs. The existence of only a single liprin- $\alpha$ gene in invertebrates makes it simpler to assess in vivo function of Liprin- $\alpha$ in the nervous system. A loss of function in syd-2 gene (Liprin- $\alpha$ homolog in Caenohabiditis elegans) alters the size and shape of presynaptic termini ${ }^{29-32}$. Deletion of the IIa RPTP homolog, ptp3 , resulted in mislocalization of SYD-2. In Drosophila, Dliprin- $\alpha$, a homolog of Liprin- $\alpha$, is required for normal synaptic morphology at neuromuscular junction and for photoreceptor target selection $^{28,33,34}$.

Although all four isoforms of Liprin- $\alpha$ are expressed in the brain with differential distribution and increase the complexity of their neuronal functions, Liprin- $\alpha 2$ and $-\alpha 3$ are predominantly and specifically expressed in the brain ${ }^{22,35,36}$. Knockdown of Liprin- $\alpha 2$ by shRNA alters synaptic vesicle pool size and presynaptic ultrastructure. Liprin- $\alpha 2$ regulates the turnover of the active zone proteins, calcium/calmodulin-dependent serine kinase (CASK) and RIM1/2, to facilitate synaptic transmission ${ }^{37}$. Superresolution microscopy revealed substantial difference in the
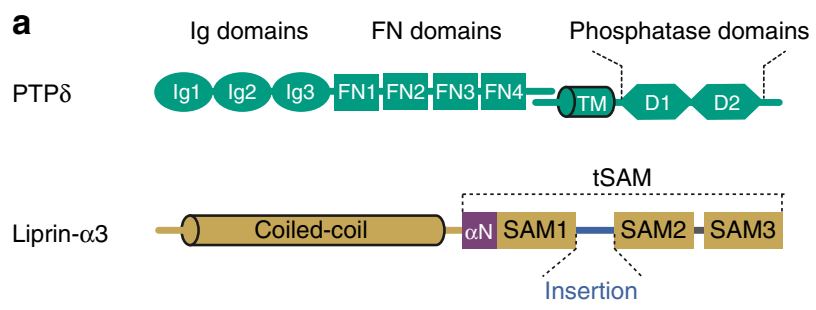

b
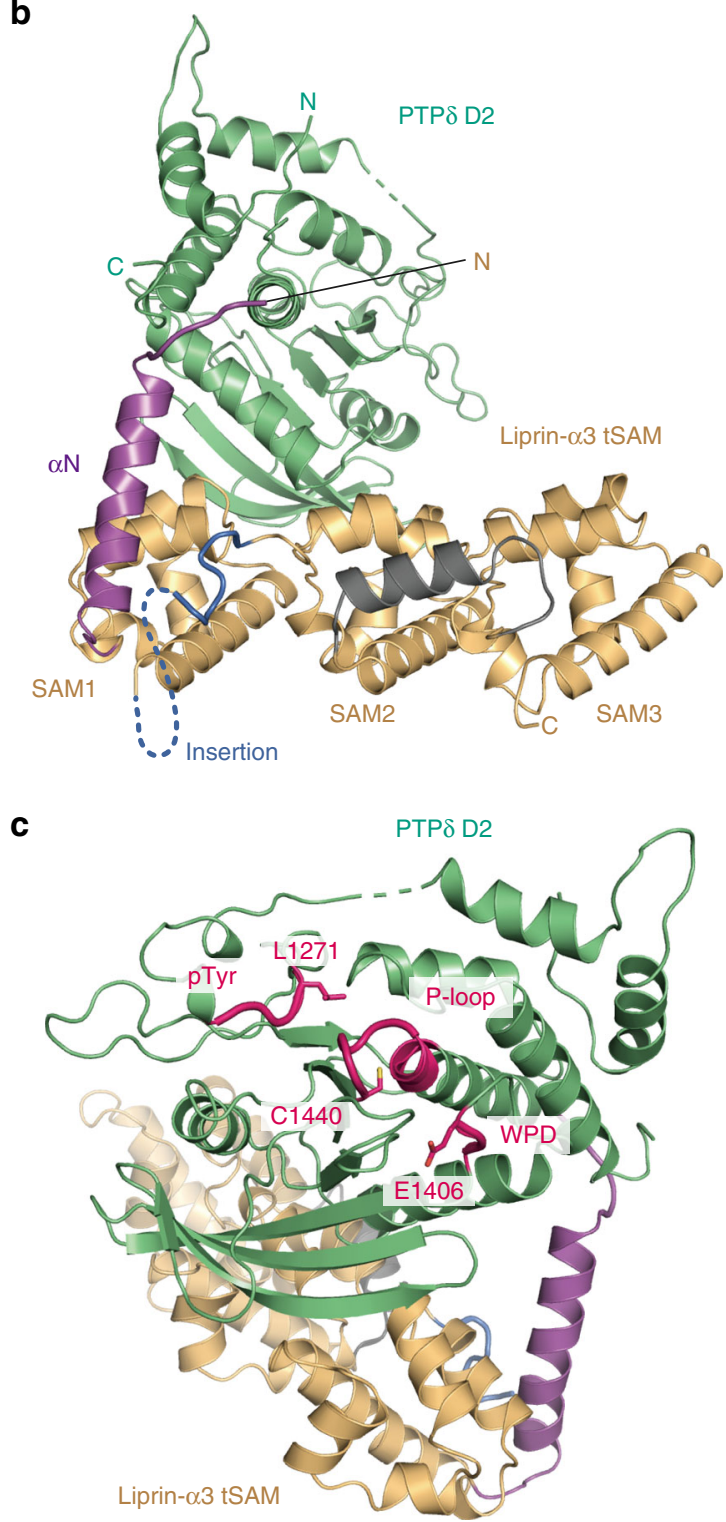

Fig. 1 Structure of the complex between PTPS D2 and Liprin- $\alpha 3$ tSAM. a Domain organizations of PTP $\delta$ and Liprin- $\alpha 3$ (Ig, Immunoglobulin-like; FN, Fibronectin type III; TM, transmembrane; SAM, sterile alpha motif; tSAM, tandem SAM). b Overall structure of the complex between PTPS D2 (green) and Liprin- $\alpha 3$ tSAM ( $\alpha \mathrm{N}$, purple; SAM1-SAM3, light brown; the insertion between SAM1 and SAM2, dark blue; the linker helix between SAM2 and SAM3, dark gray). Disordered regions are shown as dotted lines. c Pseudocatalytic site of PTPס D2. The coloring scheme is the same as that in $\mathbf{b}$, except that the pTyr, P-loop, and WPD regions are colored in magenta. 
localization of Liprin- $\alpha 2$ and $-\alpha 3$ inside the presynapse ${ }^{38}$. Liprina3 is substantially colocalized with the active zone proteins, whereas Liprin- $\alpha 2$ is localized to more internal region of the nerve terminals. Liprin- $\alpha 3$-knockout mice generated by CRISPR/ Cas9 gene editing show impaired exocytosis of synaptic vesicles, although they survive and develop mostly normally, possibly due to a complementary function of Liprin- $\alpha 2^{38}$. In fact, the depletion of Liprin- $\alpha 3$ causes the translocation of Liprin- $\alpha 2$ to the acive zone, though their functions may not be completely overlapped $^{37,38}$.

The Liprin family shares similar domain architecture comprising an N-terminal coiled-coil domain and three tandem sterile alpha motifs (SAM1-SAM3; hereafter referred to as tSAM; Fig. 1a). The N-terminal coiled-coil domain of Liprin- $\alpha$ mediates the binding to CAST/ELKS ${ }^{39}$, GIT1 ${ }^{40}, \mathrm{RIM}^{37}, \mathrm{KIF} 1 \mathrm{~A}^{41}$, and $\mathrm{mDia}^{42,43}$. The tSAM domain of Liprin- $\alpha$ interacts with the D2 domain of IIa RPTPs ${ }^{21,22}$ and CASK ${ }^{44}$. In addition, Liprin- $\alpha$ and $-\beta$ form a heterodimer through the interaction between Liprin- $\alpha$ SAM1 and Liprin- $\beta$ SAM $3^{22,45}$. A wide variety of binding partners for Liprin- $\alpha$ imply its broad synaptic functions.

The complex structure between the tSAM domain of Liprin- $\alpha 2$ and CASK revealed that the insertion loop between SAM1 and SAM2 in Liprin- $\alpha 2$ interacts with CASK ${ }^{45}$. Notably, this insertion loop is only found in the vertebrate Liprin family. In the complex structure between the tSAM domains of Liprin- $\alpha 2$ and $-\beta 1$, their heterodimeric assembly was mediated by their SAM1 and SAM3 motifs, respectively ${ }^{45}$. On the other hand, no structural information of the IIa RPTP-Liprin- $\alpha$ complex is available, although their interaction is likely to be the first important intracellular process for inducing presynaptic differentiation. Here, we present the crystal structure of the complex between PTP $\delta$ D2 and the tSAM domain of Liprin- $\alpha 3$. The D2 domain of PTP $\delta$ interacts with the N-terminal helix $(\alpha \mathrm{N})$, SAM1, and SAM2 in the tSAM domain of Liprin-a3. The structure-based mutational analyses in vitro and in cellulo demonstrate that the interaction with the SAM1 and SAM2 domains of Liprin- $\alpha 3$ is essential for the binding and synaptogenic activity.

\section{Results}

Overall structure of the PTPS D2-Liprin- $\alpha 3$ tSAM complex. To reveal the structural basis of the interaction between IIa RPTPs and Liprin- $\alpha$, we sought to determine the crystal structure of the complex between the intracellular domain of IIa RPTPs and the tSAM domain of Liprin- $\alpha$. The D1-D2 and D2 domains of mouse PTP $\delta$ and PTP $\sigma$ and the tSAM domains of mouse Liprin- $\alpha 1,-\alpha 2,-\alpha 3$, and - $\alpha 4$ were purified and subjected to cocrystallization screening. Their expression constructs were designed, based on the structures of Liprin- $\alpha 2$ tSAM $^{45}$ and the phosphatase domains of IIa RPTPs ${ }^{46}$. Consequently, the combination of PTP $\delta$ D2 and Liprin- $\alpha 3$ tSAM yielded diffractionquality crystals. The crystal structure of the PTP $\delta$ D2-Liprin- $\alpha 3$ tSAM complex was determined by the molecular replacement method using PTPo D2 (PDB 2FH7 [https://doi.org/10.2210/ pdb2FH7/pdb]) ${ }^{46}$ and Liprin-a2 tSAM (PDB 3TAD [https:// doi.org/10.2210/pdb3TAD/pdb]; the CASK-interacting loop between SAM1 and SAM2 was trimmed) ${ }^{45}$ as the search models. The asymmetric unit contains one complex, and no obvious molecular contact suggestive of higher-order assembly was found in the crystal (Supplementary Fig. 1). The final model was refined at $1.91 \AA$ resolution with $R_{\text {free }}$ of 0.208 (Table 1 and Fig. 1a, b). The residue numbering of PTP $\delta$ in this paper is based on a mouse PTP $\delta$ isoform (NM_011211.3 [https://www.ncbi.nlm.nih.gov/nuccore/NM_011211.3]), which contains four $\mathrm{FN}$ domains with the 9-residue meA and meB peptide insertions $(\mathrm{A} 9 \mathrm{~B}+)$.
Table 1 Data collection and refinement statistics.

\begin{tabular}{|c|c|}
\hline \multirow{2}{*}{\multicolumn{2}{|c|}{ Data collection }} \\
\hline & \\
\hline Beamline & SPring-8 BL41XU \\
\hline Space group & $P 4_{1} 2_{1} 2$ \\
\hline \multicolumn{2}{|l|}{ Cell dimensions } \\
\hline$a, b, c(\AA)$ & $98.0,98.0,140.0$ \\
\hline$\alpha, \beta, \gamma\left(^{\circ}\right)$ & $90.0,90.0,90.0$ \\
\hline Resolution $(\AA)$ & $50-1.91(1.94-1.91)$ \\
\hline$R_{\text {sym }}$ & $0.105(0.278)$ \\
\hline$|/ \sigma|$ & $22.1(2.3)$ \\
\hline Completeness (\%) & $97.3(91.2)$ \\
\hline Redundancy & $10.2(3.5)$ \\
\hline \multicolumn{2}{|l|}{ Refinement } \\
\hline Resolution $(\AA)$ & 1.91 \\
\hline No. reflections & $52,074(2,644)$ \\
\hline$R_{\text {work }} / R_{\text {free }}$ & $0.162 / 0.208$ \\
\hline \multicolumn{2}{|l|}{ No. atoms } \\
\hline Protein & 4,588 \\
\hline Water & 428 \\
\hline \multicolumn{2}{|l|}{$B$ factors $\left(\AA^{2}\right)$} \\
\hline Protein & 35.2 \\
\hline Water & 40.3 \\
\hline \multicolumn{2}{|l|}{ R.m.s. deviations } \\
\hline Bond lengths $(\AA)$ & 0.007 \\
\hline Bond angles $\left({ }^{\circ}\right)$ & 1.020 \\
\hline
\end{tabular}

Data were collected from a single crystal. Highest-resolution shell is in parentheses.

The D2 domain of PTP $\delta$ adopts an $\alpha / \beta$ structure, which closely resembles the reported D1 and D2 structures of IIa RPTPs (Ca rmsds of $0.59-1.23 \AA$ over 244-287 residues; Supplementary Table 1$)^{46,47}$. The pseudoactive site of PTP $\delta$ D2 retains the catalytic cysteine residue (Cys1440). However, the tyrosine residue in the phosphorylated Tyr recognition loop and the aspartate residue in the WPD motif, which are critical for the phosphatase activity ${ }^{48,49}$, are replaced with Leu and Glu, respectively. These replacements synergistically reduce the phosphatase activity ${ }^{47,50}$. The interface between Liprin- $\alpha 3$ and PTP $\delta$ D2 is located on the opposite side of the pseudoactive site (Fig. 1c)

The tSAM domain of Liprin- $\alpha 3$ consists of three SAM domains, SAM1-SAM3. The additional helix is located at the $\mathrm{N}$-terminal end of SAM1 $(\alpha \mathrm{N})$. SAM2 and SAM3 are connected by the short linker helix (Fig. 1a, b). The electron density of the CASK-interacting loop connecting SAM1 and SAM2 was invisible. The individual SAM structures and their relative configurations are similar between PTP $\delta$ D2-bound Liprin- $\alpha 3$ and CASK-bound Liprin- $\alpha 2$ (Ca rmsd of $1.39 \AA$ over 258 residues; Supplementary Table 1$)^{45}$.

Interactions between PTPS D2 and Liprin- $\mathbf{3}$ tSAM. The D2 domain of PTP $\delta$ interacts with $\alpha \mathrm{N}, \mathrm{SAM} 1$, and SAM2 of Liprina3 but not with SAM3 in the present crystal structure of the PTP $\delta$ D2-Liprin- $\alpha 3$ tSAM complex (Fig. 2a and Supplementary Table 2). At the interface with $\alpha \mathrm{N}$, Phe1503 and Tyr1506 of PTP $\delta$ hydrophobically interact with Leu808 of Liprin-a3, and Asp1504 of PTP $\delta$ forms hydrogen bonds with Arg816 of Liprin- $\alpha 3$ (Fig. 2b). At the interface with SAM1, Tyr1373, Leu1380, Phe1399, and Phe1430 of PTP $\delta$ form a hydrophobic pocket, which accommodates Trp856 of Liprin- $\alpha 3$ (Fig. 2c). At the interface with SAM2, Phe1430 of PTP $\delta$ hydrophobically interacts with Leu978 of Liprin-a3, whereas Arg1397 and Asp1433 of PTP $\delta$ form hydrogen bonds with Glu976 and Arg971 of Liprina3, respectively (Fig. 2d). Note that Phe1430 of PTPS hydrophobically interacts with both SAM1 and SAM2 of Liprin- $\alpha 3$. 

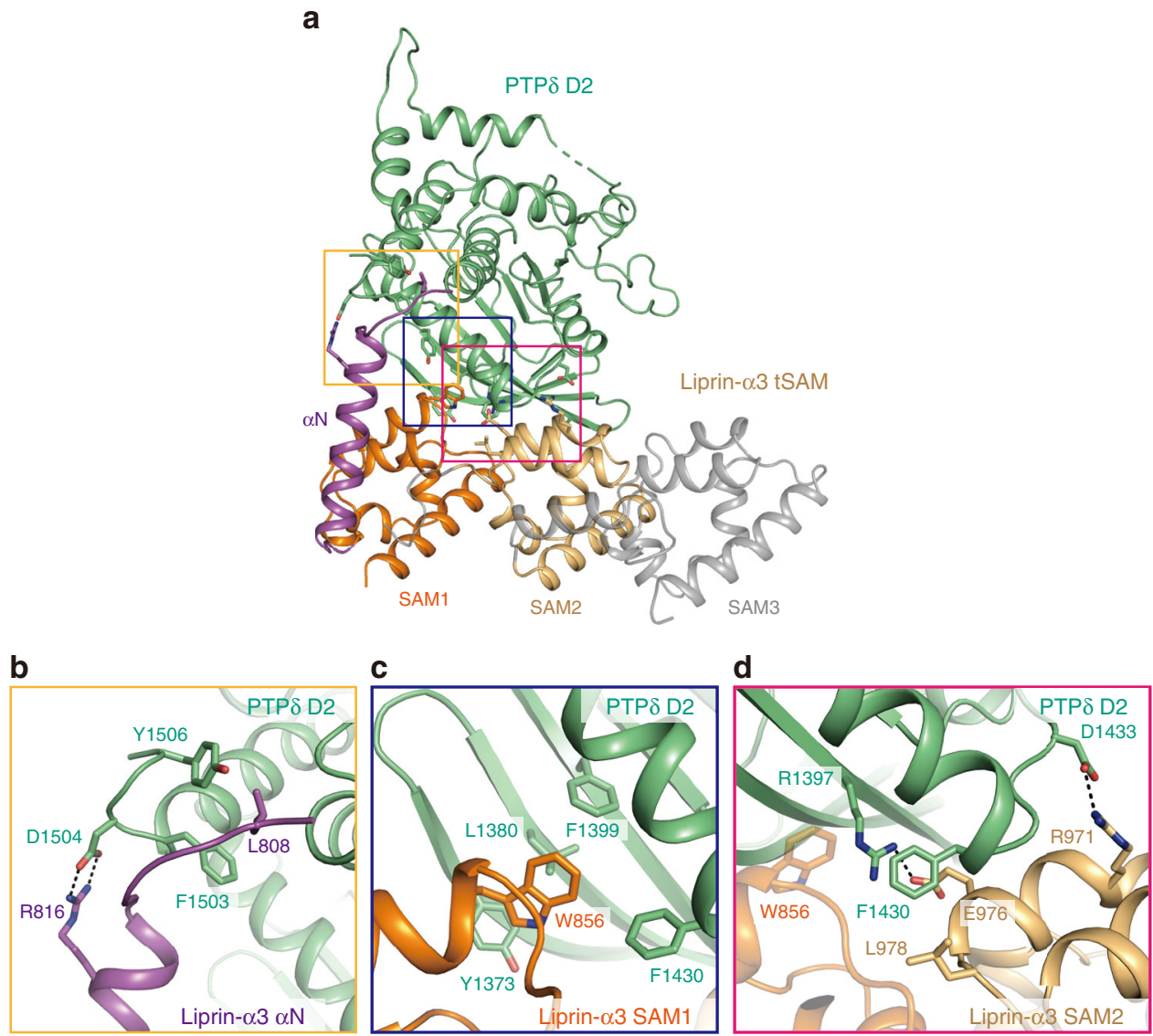

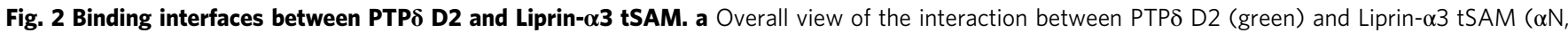
purple; SAM1, orange; SAM2, light brown; other regions, gray). Disordered regions are shown as dotted lines. The residues involved in the PTPS D2-Liprin$\alpha 3$ tSAM interaction are shown as sticks. Close-up views of the interactions of PTPס D2 with $\alpha \mathrm{N}(\mathbf{b})$, SAM1 (c), and SAM2 (d) of Liprin- $\alpha 3$ tSAM. The coloring scheme is the same as that in $\mathbf{a}$. Hydrogen bonds are shown as dotted black lines.

Table 2 Mutational analyses of the interaction between PTPס D2 and Liprin- $\alpha 3$ tSAM by SPR experiments.

\section{Mutation}

\section{Liprin- $\alpha 3$ tSAM}

WT

$\triangle \mathrm{N} 9$

L808A

R816A

W856A

E976A

L978A

PTP6 D2

F1503A

D1504A

Y1506A

Y1373A

L1380A

F1399A

R1397A

F1430A

Source data are provided as a Source Data file.

WT wild type, ND not detectable.

To assess the contribution of these intermolecular interactions to the binding affinity between PTP $\delta$ D2 and Liprin- $\alpha 3$ tSAM, site-directed mutants of PTP $\delta$ D2 or Liprin- $\alpha 3$ tSAM were analyzed by surface plasmon resonance (SPR) spectroscopy (Table 2 and Supplementary Fig. 2). Unexpectedly, point mutations or 9 -residue deletion $(\Delta N 9)$ at the interface with aN showed little effects on the affinity between PTPS D2 and Liprin- $\alpha 3$ tSAM. In contrast, mutations at the interfaces with SAM1 and SAM2 severely disrupted the binding. As for the mutations at the SAM1 interface, the W856A mutation of Liprin- $\alpha 3$ completely abolished the binding to PTP $\delta$ D2. Correspondingly, the Y1373A mutation of PTP $\delta$ almost abolished the binding, whereas the L1380A and F1399A mutations reduced the affinities $>$ fourfold and threefold, respectively. As for the mutations at the SAM2 interface, the L978A and E976A mutations of Liprin- $\alpha 3$ almost abolished the binding and reduced the affinity >fourfold, respectively. The F1430A and R1397A mutations of PTP $\delta$ both impaired the binding. Taken together, the SAM1 and SAM2 interfaces are critical for the binding between PTP $\delta$ D2 and Liprin- $\alpha 3$ tSAM, whereas the $\alpha \mathrm{N}$ interface contributes little to the binding. The key residues for the PTP $\delta$ D2-Liprin- $\alpha 3$ tSAM interaction are conserved in both IIa RPTPs and Liprin-a proteins from representative metazoa (Supplementary Figs. 3 and 4).

Mechanisms for specificity between IIa RPTP D2 and Liprin-a. The D2 domain of IIa RPTPs binds to Liprin-a proteins but not to Liprin- $\beta$ proteins ${ }^{21}$, despite their some sequence similarity (e.g., $36 \%$ identity between mouse Liprin- $\alpha 3$ SAM1-SAM2 and Liprin$\beta 1$ SAM1-SAM2; the CASK-interacting loop of Liprin- $\alpha 3$ was excluded). Superposition of the structures of mouse Liprin- $\alpha 3$ and Liprin- $\beta 1$ highlights that Trp856 and Leu978 of Liprin- $\alpha 3$, which 
are critical for binding to PTP $\delta$ and conserved in the Liprin- $\alpha$ family, are replaced with Ser and Arg in Liprin- $\beta 1$, respectively (Fig. 3a). Similar differences are found between the Liprin- $\alpha$ and Liprin- $\beta$ families (Supplementary Fig. 5), explaining the specific binding of IIa RPTPs to Liprin- $\alpha$.

A previous yeast two-hybrid assay showed that Liprin-al binds to the isolated LAR D2 but not to D1 ${ }^{21}$, although the D1 and D2 domains are topologically similar to each other ${ }^{46,47}$. To elucidate the mechanism for the specificity of Liprin- $\alpha$ to the D2 domain of IIa RPTPs, the D2 structure of PTP $\delta$ was compared with the D1 structure. Among the PTP $\delta$ D2 residues that were found to be critical for binding to Liprin- $\alpha$ in this study, Tyr1373, Leu1380, and Phe1430 of PTP $\delta$ D2 are not present in the corresponding positions of PTP $\delta$ D1 (Fig. 3b). Particularly, substantial structural difference is observed in the region around Phe1430 of PTP $\delta$ D2. At the interface between PTP $\delta$ D2 and Liprin- $\alpha 3$ SAM2, Phe1430 of PTP $\delta$ D2 is located on the edge of an $\alpha$-helix. The corresponding position in the D1 domain of IIa RPTPs is occupied with the highly conserved proline residue, which shortens the $\alpha$-helix so as to disable the interaction with Liprin- $\alpha$.

The structural mechanism for the interaction between PTP $\delta$ D2 and Liprin- $\alpha 3$ tSAM, which we revealed in this study, confirms the specific binding between the D2 domain of IIa RPTPs and the tSAM domain of Liprin- $a$ proteins.

Impact of PTP $\delta$-Liprin- $\alpha$ interaction on presynapse formation. Essential roles of Liprin- $\alpha$ in synapse formation have been demonstrated by knockout experiments of C. elegans and D. melanogaster, both of which have a single liprin- $\alpha$ gene. On the other hand, vertebrates have several Liprin- $\alpha$ isoforms (e.g., four isoforms in mouse and human), and a complete shutdown of Liprin- $\alpha$-mediated signaling likely requires a multiple gene knockout approach. In Liprin- $\alpha 3$-null mice, Liprin- $\alpha 2$ possibly compensates for the lack of Liprin- $\alpha 3$.

The present structural analysis of the interaction between PTP $\delta$ D2 and Liprin- $\alpha 3$ tSAM unveiled the critically important residues of IIa RPTPs for binding to Liprin- $\alpha$ proteins. The mutations of these residues may selectively shutdown the PTP $\delta$-Liprin- $\alpha$ signaling axis for inducing presynaptic differentiation. To test this possibility, we examined the effects of the PTP $\delta$ mutations that impair the interaction with either or both SAM1 and SAM2 domains of Liprin- $\alpha$ on presynaptic differentiation. Among postsynaptic organizer proteins that induce presynaptic a
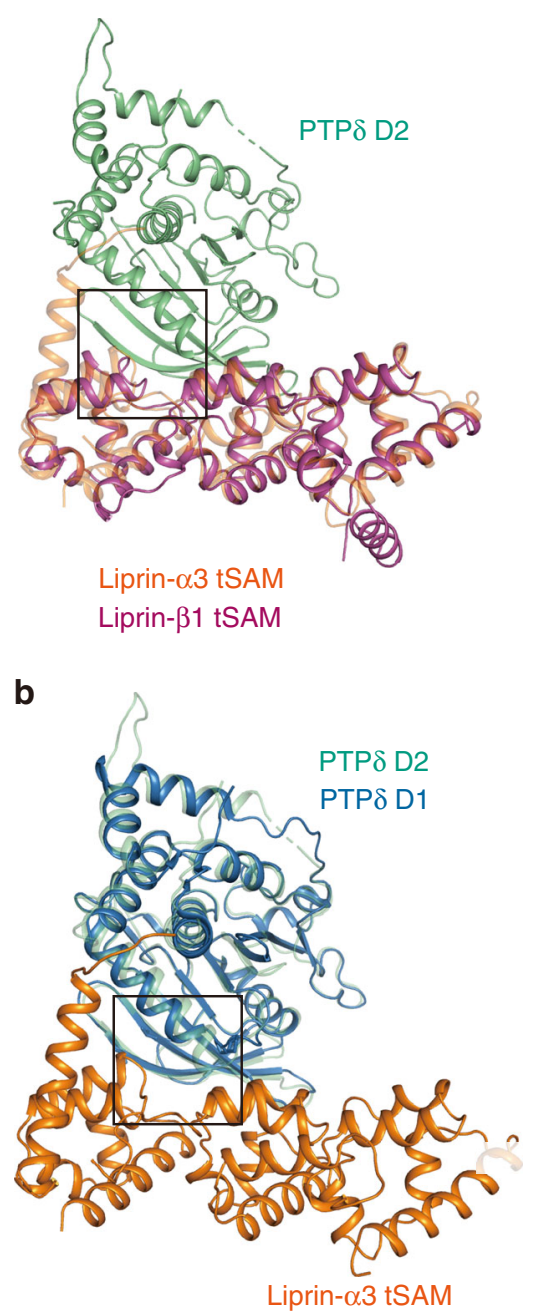
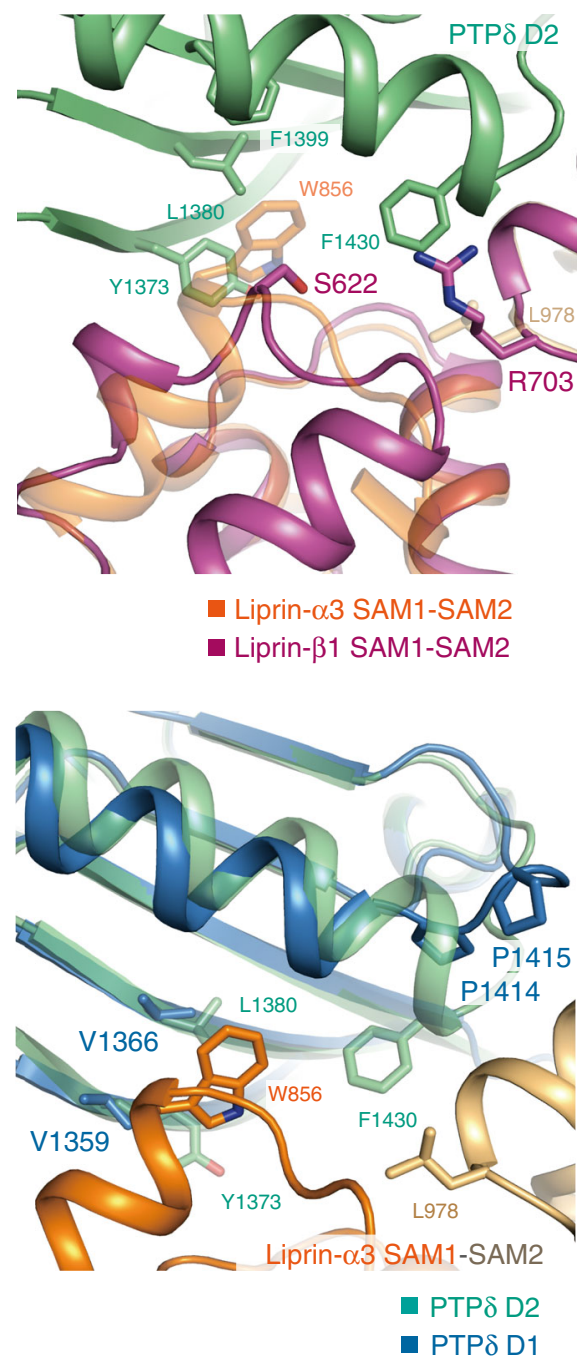

Fig. 3 Structural comparisons between Liprin- $\boldsymbol{\alpha}$ and $\mathbf{- \beta}$ and between PTPס D2 and D1. a Superposition of Liprin- $\beta 1$ (purple) on Liprin- $\alpha 3$ (orange) bound

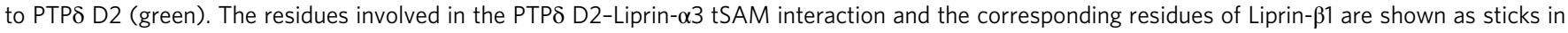
the right panel. b Superposition of PTPS D1 (blue) on PTPS D2 (green) bound to Liprin- $\alpha 3$ tSAM (SAM1, orange; SAM2, light brown). The residues involved in the PTPS D2-Liprin- $\alpha 3$ tSAM interaction and the corresponding residues of PTPS D1 are shown as sticks in the right panel. 
differentiation through IIa RPTPs, we focused on IL1RAPL1 as an inducer because its synaptogenic activity is entirely dependent on the interaction with presynaptic $\mathrm{PTP} \delta^{10}$. The IL1RAPL1-PTP $\delta$ interaction requires two short peptide inserts (meA-peptide and meB-peptide) within the Ig-like domains of PTPS, which are derived from mini-exons A and B of Ptprd gene encoding PTP $\delta$ protein ${ }^{10,18}$. We then generated a mutant mouse line that lacks meB-peptide-containing PTP $\delta$ splice variants capable of binding to IL1RAPL1 by inserting a stop codon into mini-exon B (exon 9) of Ptprd gene. The Ptprd allele with the mini-exon B mutation is referred to as $\operatorname{Ptprd}^{m e B^{*}}$ in this study.
When cocultured with wild-type cortical neurons, beads conjugated with the recombinant extracellular domain of IL1RAPL1 strongly induced the accumulation of presynaptic reporter proteins, synaptophysin-mCherry and EGFP-Rab3, in contacting axons (Fig. 4a). However, as expected, cultured cortical neurons from the homozygous mutant mice $\left(P t p r d^{m e B^{*} / m e B^{*}}\right)$ completely lacked the presynapse-inducing activity (Fig. $4 \mathrm{~b}, \mathrm{c}$ ). Therefore, we performed add-back experiments, in which cortical neurons from the Ptprd ${ }^{m e B^{*} / m e B^{*}}$ mice were transfected with wild-type or mutated forms of PTP $\delta$ linked to EGFP-Rab3 by a self-cleaving P2A peptide and incubated with the a IL1RAPL1
-Fc beads

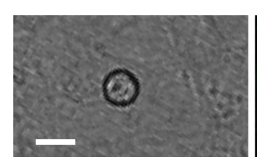

b

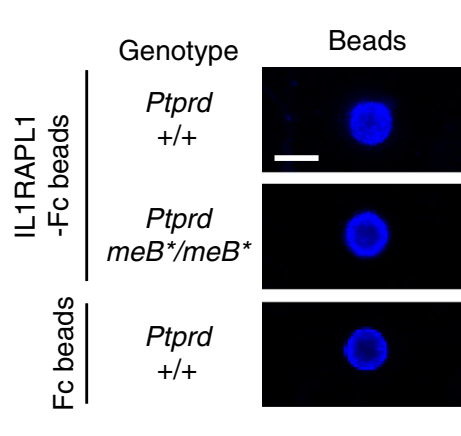

d

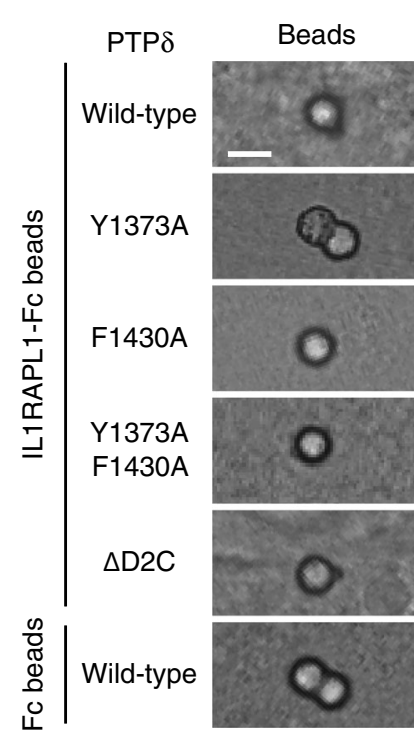

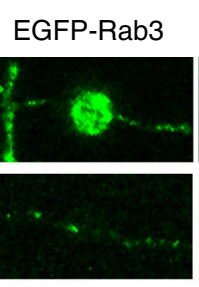
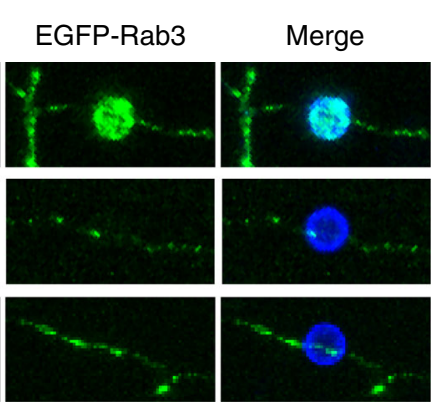

Synaptophysin
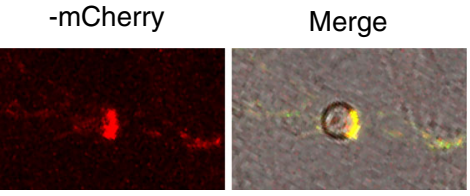

C Presynapse-inducing activity

(EGFP-Rab3 signals)

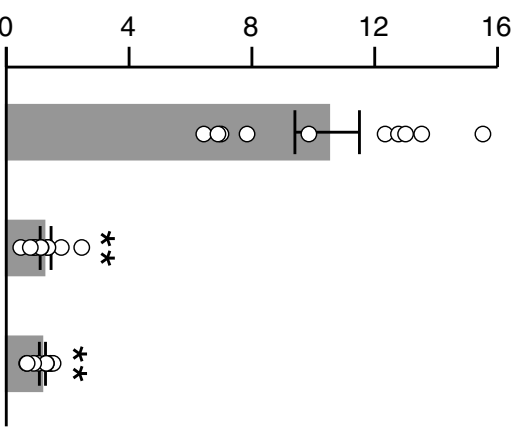

$\mathbf{e}$

Presynapse-inducing activity (EGFP-Rab3 signals)
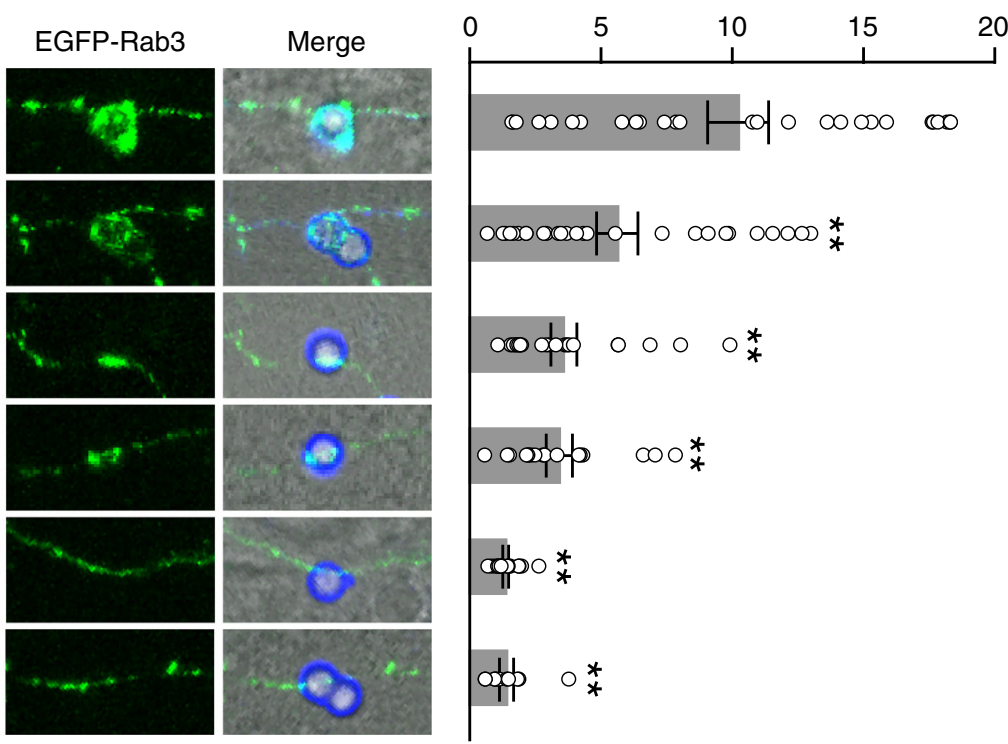

Fig. 4 Synaptogenic activities of PTPS mutants impairing Liprin interaction. a IL1RAPL1-induced presynaptic differentiation of wild-type cortical neurons monitored by accumulation of EGFP-Rab3 (green) and synaptophysin-mCherry (red). b Complete loss of presynapse-inducing activity of IL1RAPL1 in cortical neurons from Ptprdme $B^{\star} /$ me $^{\star}$ mutant mice. c Relative intensity of EGFP-Rab3 signals on the surface of beads in $\mathbf{b}$ ( $n=10$ beads each). $\mathbf{d}$ Rescue of IL1RAPL1-induced EGFP-Rab3 accumulation in Ptprdme $B^{\star} / m e B^{\star}$ cortical neurons by transient expression of wild-type and mutated forms of PTP8. e Relative intensity of EGFP-Rab3 signals on the surface of beads in $\mathbf{d}$ ( $n=27,27,22,17$, and 18 IL1RAPL1-Fc beads for wild type, Y1373A, F1430A, Y1373A/F1430A, and $\triangle \mathrm{D} 2 \mathrm{C}$, respectively; $n=11 \mathrm{Fc}$ beads for wild type). All values represent mean \pm SEM. ${ }^{\star \star} P<0.01$ compared with wild-type neurons with IL1RAPL1 beads in $\mathbf{b}$ and compared with neurons rescued by wild-type PTP $\delta$ and cocultured with IL1RAPL1 beads in d; Tukey's test. Scale bars, $5 \mu \mathrm{m}$. Source data are provided as a Source Data file. 
IL1RAPL1-conjugated beads (Fig. 4d, e). For this rescue experiments, the D2 domain mutations at the interface with Liprin- $\alpha$ were introduced into the PTP $\delta$ splice variant with both meA-peptide and meB-peptide insertions, which shows the highest affinity to IL1RAPL 18 . Add-back of wild-type PTP $\delta$ restored the IL1RAPL1-induced presynaptic differentiation (i.e., accumulation of EGFP-Rab3), while deletion of the D2 domain and C-terminal tail of PTP $\delta(\triangle \mathrm{D} 2 \mathrm{C})$ completely lacked the ability to rescue the defect in the presynaptic differentiation (Fig. $4 \mathrm{~d}, \mathrm{e}$ ), suggesting that the D2 domain is required for synaptogenesis induced by PTP $\delta$ as well as by $\mathrm{PTP}^{25,51}$. The triple mutant of PTP $\delta$ for the interface with Liprin- $\alpha$ aN (F1503A D1504A Y1506A) restored presynaptic differentiation like wild type, in agreement with the fact that $\alpha \mathrm{N}$ does not contribute to the binding to PTP $\delta$ D2 (Supplementary Fig. 6). On the other hand, the single point mutations of PTP $\delta$ at the interface with Liprin-a SAM1 (Y1373A) or SAM1/SAM2 (F1430A) disturbed the rescue activity (Fig. $4 \mathrm{~d}$, e), suggesting the importance of the PTP $\delta$-Liprin- $\alpha$ interactions through the SAM1 and SAM2 domains for PTP $\delta$ to induce presynaptic differentiation. Tyr1373 contributes exclusively to the SAM1 interface, whereas Phe1430 does mainly to the SAM2 interface. The SAM2 interface might be more important for synaptogenic activity than the SAM1 interface. Unexpectedly, the Y1373A F1430A double mutant of PTP $\delta$ retained synaptogenic activity comparable with the F1430A mutant (Fig. 4d, e). The F1430A mutation has a larger impact than the Y1373A mutation, and may mask the impact of the Y1373A mutation in a way analogous to two factors in a linear
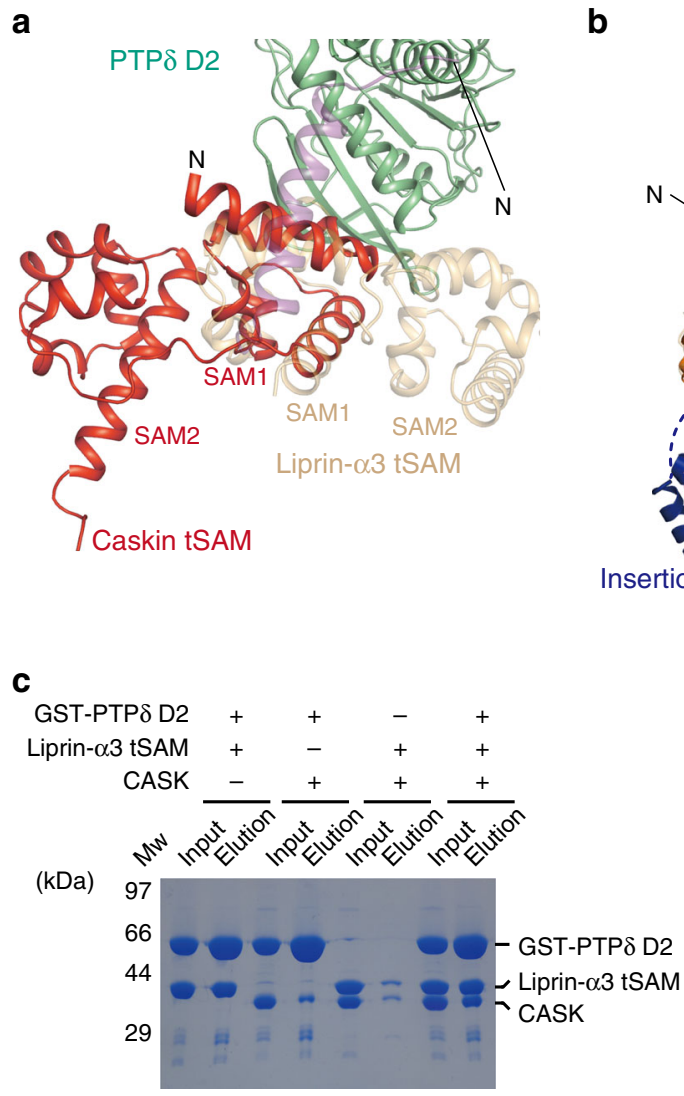

Fig. 5 Structure-based assessment of the involvement of Caskin and CASK. a Superposition of Caskin SAM1-SAM2 (red) on Liprin- $\alpha 3$ tSAM ( $\alpha$ N, purple; SAM1-SAM2, light brown) bound to PTPS D2 (green). b Superposition of the CASK (pink)-Liprin- $\alpha 2$ tSAM (CASK-interacting loop, dark blue; other regions, orange) complex on the PTP D D (green)-Liprin- $\alpha 3$ tSAM (light brown) complex. No steric hindrance in this superposition suggests the formation of the tripartite signaling complex of PTPS, Liprin- $\alpha$, and CASK. c GST-pulldown assay for analyzing the formation of the tripartite complex suggested in b. Proteins bound to GST-PTPS D2 were subjected to SDS-PAGE and stained by Coomassie Brilliant Blue. Source data are provided as a Source Data file.

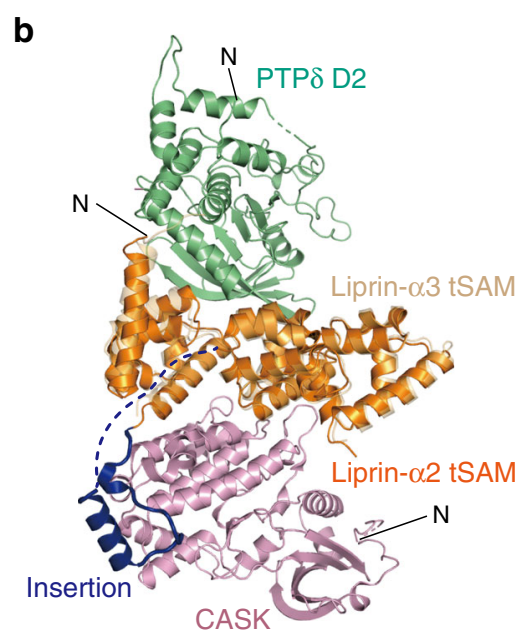

pathway. Otherwise, there may be another signaling pathway not mediated by Liprin- $\alpha$.

\section{Discussion}

In this study, we clarified the binding mode between PTP $\delta$ D2 and Liprin- $\alpha$ tSAM. Caskin is a synaptic protein, which was first identified as a CASK-binding protein from rat brain extracts. Caskin has an N-terminal ankyrin repeat domain, SH3 domain, and tSAM domain consisting of two SAM domains, SAM1 and SAM2, and is assumed to serve as an adapter molecule in synapses $^{52}$. It has been reported that the tSAM domain of Caskin2 binds to the D2 domain of LAR and PTP ${ }^{23}$. However, the relative configuration of SAM1 and SAM2 are obviously different between Caskin-1 and Liprin- $\alpha 3$. When superposing the tSAM domain of Caskin-1 (PDB 3SEI [https://doi.org/10.2210/pdb3SEI/ pdb] $)^{52}$ onto that of Liprin- $\alpha 3$ bound to PTP $\delta$ D2 using SAM1 as the reference, the position of the SAM2 domain is completely different between Caskin-1 and Liprin- $\alpha 3$ (Fig. 5a). Therefore, the tSAM domain of Caskin-1 binds to the D2 domain of LAR and $\mathrm{PTP} \sigma$, likely in a manner different from the tSAM domain of Liprin- $\alpha$.

The tSAM domain of Liprin- $\alpha$ can also bind to CASK. The insertion loop connecting the SAM1 and SAM2 domains is essential for this binding, and the isolated loop itself can bind to $\mathrm{CASK}^{45}$. The PTP $\delta$ D2-binding surface of Liprin-a3 tSAM is located on the opposite side of the insertion loop. When superposing the CASK-Liprin- $\alpha 2$ tSAM structure (PDB 3TAC [https://doi.org/10.2210/pdb3TAC/pdb]) onto the present PTPS 
D2-Liprin- $\alpha 3$ tSAM structure using Liprin- $\alpha$ tSAM as the reference, no steric hindrance occurs between CASK and PTP $\delta$ D2 (Fig. 5b). In addition, further docking of D1 on the basis of the superposition of the PTP $\delta$ D2-Liprin- $\alpha 3$ tSAM complex onto PTP $\sigma$ D1-D2 (PDB 2FH7 [https://doi.org/10.2210/pdb2FH7/pdb]) using D2 as the reference suggests that D1 does not overlap with Liprin-a3 tSAM or CASK (Supplementary Fig. 7a). These docking analyses suggest the tripartite assembly of IIa RPTP, Liprin- $\alpha$, and CASK. This idea was supported by GST-pulldown assay, where Liprin- $\alpha 3 \mathrm{tSAM}$ and the N-terminal kinase domain of CASK were co-precipitated with the GST-fused PTP $\delta$ D2 or D1D2 (Fig. 5c and Supplementary Fig. 7b). The predicted geometry of the tripartite IIa RPTP-Liprin-a-CASK complex is compatible to the idea that this complex can be further assembled into other important presynaptic proteins such as $\mathrm{RIM}^{37}, \mathrm{mDia}^{42,43}$, Liprin$\beta^{22,45}$, and GRIP1 ${ }^{53}$. Such higher-order assembly of intracellular presynaptic proteins might be functionally and mechanistically linked to lateral alignments of transsynaptic connections via synaptic organizers ${ }^{16,54}$. Further studies on the mechanism for spatiotemporal regulation of this assembly may deepen our understanding of the molecular mechanism for synapse formation.

\begin{abstract}
Methods
Cloning and plasmid construction. For the structural and SPR analyses, the genes encoding mouse PTP $\delta$ D2 (residues 1213-1508) and mouse Liprin- $\alpha 3$ tSAM (residues 808-1114) were PCR amplified from cDNAs and cloned into the pET21a (Millipore) and pGEX-6P1 vectors (GE Healthcare), respectively. For pull-down assay, the genes encoding mouse CASK N-terminal kinase domain (residues $1-323$ ) was cloned into the pET21a vector. PTP $\delta$ D2 (residues 1213-1508) and D1D2 (residues 891-1508) were cloned into the pGEX-6P1 vector. For synaptogenic assay, the coding sequences of preprotrypsin signal peptide followed by FLAG tag from pFLAG-CMV-1 vector (Sigma), mouse PTP $\delta$ lacking signal peptide, porcine teschovirus-1 P2A peptide 55 , EGFP, and mouse Rab3 were linked and cloned into pCAGGS vector ${ }^{56}$ to yield pCAGGS-ppt-FLAG-PTPRD-P2A-EGFP-Rab3. The alanine substitution mutations within D2 domain were introduced by inverse PCR mutagenesis using pCAGGS-ppt-FLAG-PTPRD-P2A-EGFP-Rab3 as a template. The coding sequences of mouse synaptophysin and Rab3 were cloned into pEGFP$\mathrm{C} 1$ (Clontech) and pmCherry-N1 (Clontech) vectors, respectively.
\end{abstract}

Protein preparation. All proteins were expressed in Escherichia coli Rosetta (DE3) cells (Millipore). The cells were disrupted by sonication. For the purification of PTPS D2, the cell lysate containing PTP $\delta$ D2 fused with the C-terminal His ${ }_{6}$-tag (PTP $\delta$ D2-His ${ }_{6}$ ) was applied onto a Ni-NTA (Qiagen) column. After washing with $20 \mathrm{mM}$ Tris-HCl buffer (pH 8.0) containing $300 \mathrm{mM} \mathrm{NaCl}$ and $50 \mathrm{mM}$ imidazole, PTP $\delta$ D2-His ${ }_{6}$ was eluted with $20 \mathrm{mM}$ Tris- $\mathrm{HCl}$ buffer ( $\mathrm{pH}$ 8.0) containing $300 \mathrm{mM} \mathrm{NaCl}$ and $250 \mathrm{mM}$ imidazole. For crystallography, PTP $\delta$ D2-His ${ }_{6}$ was further purified by size-exclusion chromatography using Superdex 200 (GE Healthcare) column with $20 \mathrm{mM}$ Tris-HCl buffer (pH 7.5) containing $150 \mathrm{mM}$ $\mathrm{NaCl}$. For the purification of Liprin- $\alpha 3$ tSAM, the cells expressing Liprin- $\alpha 3$ tSAM fused with the N-terminal glutathione-S-transferase (GST-Liprin- $\alpha 3$ tSAM) was disrupted by sonication. The cell lysate was applied onto a Glutathione Sepharose FF column (GE Healthcare). After washing by phosphate-buffered saline (PBS), GST-Liprin- $\alpha 3$ was eluted with $50 \mathrm{mM}$ Tris-HCl buffer ( $\mathrm{pH}$ 8.0) containing 200 $\mathrm{mM} \mathrm{NaCl}$ and $15 \mathrm{mM}$ reduced glutathione. The eluted sample was further purified by anion-exchange chromatography using a Resource Q column (GE Healthcare), which was pre-equilibrated with $20 \mathrm{mM}$ Tris- $\mathrm{HCl}$ buffer ( $\mathrm{pH}$ 7.5) containing $50 \mathrm{mM} \mathrm{NaCl}$. GST-Liprin- $\alpha 3$ was eluted with a linear gradient of $50-500 \mathrm{mM}$ $\mathrm{NaCl}$. The GST tag was cleaved off by HRV3c protease and removed by a Glutathione Sepharose FF column. The sample was purified again by anion-exchange chromatography in the same manner as GST-Liprin- $\alpha 3$. Finally, the sample was purified by size-exclusion chromatography using a Superdex 200 (GE Healthcare) column with $20 \mathrm{mM}$ Tris- $\mathrm{HCl}$ buffer (pH 7.5) containing $150 \mathrm{mM} \mathrm{NaCl}$.

For pull-down assay, PTP $\delta$ D2 or D1-D2 fused with an N-terminal GST tag was purified using a Glutathione Sepharose FF column, followed by size-exclusion chromatography using a Superdex 200 (GE Healthcare) column or anion-exchange chromatography using a Resource Q (GE Healthcare) column, respectively. The CASK N-terminal kinase domain fused with the $\mathrm{N}$-terminal $\mathrm{His}_{6}$-tag ( $\mathrm{His}_{6}$-CASK) was purified by Ni-affinity chromatography in the same manner as PTP $\delta$ D2-His 6 . $\mathrm{His}_{6}$-CASK was further purified by anion-exchange chromatography using a HiTrap Q HP or Resource Q column (GE Healthcare).

Crystallization and structure determination. For crystallization of the PTP $\delta$ D2-Liprin- $\alpha 3$ tSAM complex, PTP $\delta$ D2-His $6(90 \mu \mathrm{M})$ and Liprin- $\alpha 3$ tSAM
$(70 \mu \mathrm{M})$ were mixed. Initial crystallization screening was performed using the sitting drop vapor diffusion method at $20^{\circ} \mathrm{C}$ with a Mosquito liquid-handling robot (TTP Lab Tech). About 500 conditions were tested with crystallization reagent kits supplied by Hampton Research and Qiagen. After optimization of the crystallization condition, diffraction-quality crystals of the complex were obtained with the reservoir solution containing 15\% PEG 3350 and $0.1 \mathrm{M}$ Tris- $\mathrm{HCl}$ (pH 8.5). The crystals were soaked in the reservoir solution supplemented with 30\% PEG 400 and then flash-frozen in liquid $\mathrm{N}_{2}$

Diffraction data sets were collected at $100 \mathrm{~K}$ at BL41XU in SPring-8 and processed with HKL $2000^{57}$ and the CCP4 program suite ${ }^{58}$. The structure of the PTP $\delta$ D2-Liprin- $\alpha 3$ tSAM complex was determined by molecular replacement with the program Molrep ${ }^{59}$ using PTP $\sigma$ D2 (PDB 2FH7[https://doi.org/10.2210/ pdb2FH7/pdb]) $)^{46}$ and Liprin- 22 tSAM (PDB 3TAD[https://doi.org/10.2210/ $\mathrm{pdb} 3 \mathrm{TAD} / \mathrm{pdb}])^{45}$ as the search models. The atomic model was manually improved using the program $\operatorname{Coot}^{60}$, and refined using the program Phenix ${ }^{61}$ with good stereochemistry $(97.1 \%$ and $0.2 \%$ of residues in favored and outliers, respectively). Data collection and refinement statistics are summarized in Table 1 The buried surface area was calculated using the program PISA ${ }^{62}$. Sequence alignment was performed using ClustalX software ${ }^{63}$. All structure figures were prepared using the program PyMOL (Schrödinger, LLC; https://pymol.org/2/).

SPR analysis. SPR experiments were performed using Biacore T200 (GE Healthcare) at $25^{\circ} \mathrm{C}$ in $10 \mathrm{mM}$ Hepes- NaOH buffer (pH 7.5) containing $150 \mathrm{mM}$ $\mathrm{NaCl}$, and $0.005 \%$ Tween-20. Wild-type or mutant PTP $\delta$ D2-His ${ }_{6}$ was immobilized on a CM5 sensor chip by the amine-coupling method. The amount of the immobilized ligand for each experiment is shown in response units in Table 2. The wild-type or mutant Liprin- $\alpha 3$ tSAM was prepared in a twofold serial dilution series from $40 \mu \mathrm{M}$ to $39 \mathrm{nM}$. Each sample was injected in order of increasing concentration for $120 \mathrm{~s}$ at a flow rate of $30 \mu \mathrm{L} \mathrm{min}^{-1}$, followed by a 600 -s dissociation phase. The PTP $\delta$ D2-immobilized sensor chip was regenerated by $20 \mathrm{mM}$ Hepes- $\mathrm{NaOH}$ buffer ( $\mathrm{pH} 7.5$ ) containing $1 \mathrm{M} \mathrm{NaCl}$. All mutants examined by SPR analysis were confirmed to behave as wild type in size-exclusion chromatography (Supplementary Fig. 8). Equilibrium dissociation constants $\left(K_{\mathrm{D}}\right)$ were calculated using Biacore T200 software. Data are shown as mean \pm standard deviation from at least three independent experiments for each sample.

Pull-down assay. For Fig. 5c, Liprin- $\alpha 3$ tSAM and/or His $_{6}$-CASK were mixed with GST-PTP $\delta 2$ at an equimolar ratio $(20 \mu \mathrm{M})$ in PBS with $0.1 \%$ Triton X-100 (PBST), and then immobilized onto Glutathione Sepharose FF (GE Healthcare) beads The beads were washed with PBS-T three times. The bound protein complexes were then eluted with $50 \mathrm{mM}$ Tris- $\mathrm{HCl}$ buffer $(\mathrm{pH} 8.0)$ containing $200 \mathrm{mM} \mathrm{NaCl}$ and $15 \mathrm{mM}$ reduced glutathione. The eluted sample was separated from beads by centrifuge and subjected to SDS-PAGE analysis with Coomassie brilliant blue staining. For Supplementary Fig. 7, GST-PTP $\delta$ D2 or D1-D2 were immobilized onto Glutathione Sepharose FF (GE Healthcare) beads (10 $\mu \mathrm{g}$ of protein per $1 \mu \mathrm{L}$ beads) and then, mixed with $13 \mu \mathrm{M} \mathrm{His}_{6}$-CASK in PBS-T with or without $29 \mu \mathrm{M}$ Liprin- $\alpha 3$ tSAM. The beads were washed with PBS-T twice. The bound protein complexes were then eluted with 2 xSDS loading buffer and subjected to SDS-PAGE analysis with Coomassie brilliant blue staining.

Generation of Ptprd ${ }^{\text {meB }}{ }^{\star}$ mice. The electroporation of guide RNA, Cas9 protein, and ssODN into $\mathrm{C} 57 \mathrm{BL} / 6 \mathrm{~N}$ mouse zygotes was performed as previously described $^{64}$. The sequences of guide RNA (gRNA-meB) and ssODN (meB*-ssODN) were $5^{\prime}$-CAUCCCUCAGAGCUGCGAGA- ${ }^{\prime}$ and, $5^{\prime}$-CCTTTTCCTCATTTCATTGTG TTCTGCATCAAACCCCCCTACATCCCTCAGAGCTCTGAGAAGGTTGGT GTGTTTTTTACTTTTTACCCACCTTTACAAAACTACTACTT-3', respectively. The genomic DNAs from the F0 mice were subjected to cleaved amplified polymorphic sequence analyses using primer set, $5^{\prime}$-ATGGTGACCTCCTTTGCTG-3 and $5^{\prime}$-TCATGCATTGCATTTGGACG-3', and restriction enzyme SacI for the detection of the ssODN-mediated knock-in of the stop codon into meB. The F0 mosaic mice were crossed with wild-type C57BL/6N mice to generate heterozygous $\left(\right.$ Ptprd $\left.^{+/ m e B^{*}}\right)$ F1 mice. The strain was maintained by crossing $P t p r d^{+/ m e B^{*}}$ females with wild-type C57BL/6N mice. The F3 heterozygous male and female mice were mated to generate homozygous mutant mice for preparation of neuronal cultures. All experimental protocols for animal studies were approved by the Animal Experiment Committee of the University of Toyama (Authorization No. A2016med-140) and conducted in accordance with the Guidelines for the Care and Use of Laboratory Animals of the University of Toyama.

Synaptogenic assay. Cerebral cortical neurons were prepared from homozygous $\left(\right.$ Ptprd $\left.^{m e B^{*} / m e B^{*}}\right)$ mutant mice at embryonic day 18 as previously described ${ }^{9}$. The cultured neurons were transfected with expression vectors for wild-type or mutated forms of PTP $\delta$ linked to EGFP-Rab3 by P2A peptide at days in vitro (DIV) 8 , and cocultured with Fc- or IL1RAPL1-Fc-coated magnetic beads at DIV9. The cocultures were fixed and mounted for confocal microscopy at DIV10.

Image acquisition and quantification. Images of cocultures were collected from at least two separate experiments using a confocal microscope (TCS SP5II, Leica Microsystems; $63 \times$ water lens, zoom setting 3.0 ) in a blinded manner with regard 
to the expression vectors transfected. Fc- or IL1RAPL1-coated beads on the EGFPRab3-expressing axons were randomly imaged. $\mathrm{Z}$ series optical sections were projected by the brightest point method and the accumulation of EGFP-Rab3 around the beads were quantified using ImageJ software ${ }^{65}$. The axonal EGFP-Rab3 signal intensity of the bead-contacting area was measured as a fluorescent mean density within a circle of $7-\mu \mathrm{m}$ diameter enclosing the beads while averaged fluorescent mean density within $7-\mu \mathrm{m}$-diameter circles on the same axon apart from the beads was measured as a background axonal signal. Ratios of the EGFPRab3 fluorescent signal intensities of the bead-contacting region and background region of the same axon were calculated for Fig. 4. Data represent mean \pm SEM. Statistical significance was evaluated by one-way ANOVA followed by Tukey's post hoc test.

Statistical analysis. Two independent experiments were included in the statistical analysis for Fig. 4. All statistical analyses were performed using Statcel2 (OMS Publishing). No statistical method was used to determine sample size. No data were excluded. There was no randomization of mice or samples before analysis, and the mice used in this study were selected based purely on availability. For multiple comparisons, one-way ANOVA followed by Tukey's post hoc tests was used (Fig. $4 \mathrm{c}$, df numerator $(\mathrm{dfn})=2$, df denominator $(\mathrm{dfd})=27, F$ value $=76.49$; Fig. $4 \mathrm{e}, \mathrm{dfn}=5$, dfd $=116, F$ value $=17.95$; Supplementary Fig. $6 \mathrm{~b}, \mathrm{dfn}=2, \mathrm{dfd}=$ $113, F$ value $=35.98$ )

Reporting summary. Further information on research design is available in the Nature Research Reporting Summary linked to this article.

\section{Data availability}

Data supporting the findings of this manuscript are available from the corresponding authors upon reasonable request. The coordinates and structure factors of the PTP $\delta$ D2-Liprin- $\alpha 3$ tSAM complex have been deposited in the Protein Data Bank with the accession code $6 \mathrm{KIP}$ [https://doi.org/10.2210/pdb6KIP/pdb]. The source data underlying Table 2, Figs. $4 \mathrm{a}-\mathrm{e}$, and $5 \mathrm{c}$, and Supplementary Figs. $6 \mathrm{~b}$ and $7 \mathrm{~b}$ are provided as a Source Data file. Other data are available from the corresponding authors upon reasonable request.

Received: 23 July 2019; Accepted: 15 January 2020; Published online: 31 January 2020

\section{References}

1. Han, K. A., Jeon, S., Um, J. W. \& Ko, J. Emergent synapse organizers: LARRPTPs and their companions. Int. Rev. Cell Mol. Biol. 324, 39-65 (2016)

2. Sudhof, T. C. Synaptic neurexin complexes: a molecular code for the logic of neural circuits. Cell 171, 745-769 (2017).

3. Takahashi, H. \& Craig, A. M. Protein tyrosine phosphatases PTP $\delta$, РTP 6 , and LAR: presynaptic hubs for synapse organization. Trends Neurosci. 36, 522-534 (2013).

4. Um, J. W. \& Ko, J. LAR-RPTPs: synaptic adhesion molecules that shape synapse development. Trends Cell Biol. 23, 465-475 (2013).

5. Coles, C. H., Jones, E. Y. \& Aricescu, A. R. Extracellular regulation of type IIa receptor protein tyrosine phosphatases: mechanistic insights from structural analyses. Semin. Cell Dev. Biol. 37, 98-107 (2015).

6. Woo, J. et al. Trans-synaptic adhesion between NGL-3 and LAR regulates the formation of excitatory synapses. Nat. Neurosci. 12, 428-437 (2009).

7. Kwon, S. K., Woo, J., Kim, S. Y., Kim, H. \& Kim, E. Trans-synaptic adhesions between netrin-G ligand-3 (NGL-3) and receptor tyrosine phosphatases LAR, protein-tyrosine phosphatase $\delta(\mathrm{PTP} \delta)$, and PTP $\sigma$ via specific domains regulate excitatory synapse formation. J. Biol. Chem. 285, 13966-13978 (2010).

8. Takahashi, H. et al. Postsynaptic TrkC and presynaptic PTP $\sigma$ function as a bidirectional excitatory synaptic organizing complex. Neuron 69, 287-303 (2011).

9. Yoshida, T. et al. IL-1 receptor accessory protein-like 1 associated with mental retardation and autism mediates synapse formation by trans-synaptic interaction with protein tyrosine phosphatase $\delta$. J. Neurosci. 31, 13485-13499 (2011).

10. Yoshida, T. et al. Interleukin-1 receptor accessory protein organizes neuronal synaptogenesis as a cell adhesion molecule. J. Neurosci. 32, 2588-2600 (2012).

11. Takahashi, $\mathrm{H}$. et al. Selective control of inhibitory synapse development by Slitrk3-PTP $\delta$ trans-synaptic interaction. Nat. Neurosci. 15, 389-398 (2012). S1-S2.

12. Yim, Y. S. et al. Slitrks control excitatory and inhibitory synapse formation with LAR receptor protein tyrosine phosphatases. Proc. Natl Acad. Sci. USA 110, 4057-4062 (2013).

13. Li, Y. et al. Splicing-dependent trans-synaptic SALM3-LAR-RPTP interactions regulate excitatory synapse development and locomotion. Cell Rep. 12, $1618-1630$ (2015).
14. Choi, Y. et al. SALM5 trans-synaptically interacts with LAR-RPTPs in a splicing-dependent manner to regulate synapse development. Sci. Rep. 6 , 26676 (2016)

15. Coles, C. H. et al. Structural basis for extracellular cis and trans RPTPo signal competition in synaptogenesis. Nat. Commun. 5, 5209 (2014).

16. Um, J. W. et al. Structural basis for LAR-RPTP/Slitrk complex-mediated synaptic adhesion. Nat. Commun. 5, 5423 (2014).

17. Yamagata, A. et al. Structure of Slitrk2-PTP $\delta$ complex reveals mechanisms for splicing-dependent trans-synaptic adhesion. Sci. Rep. 5, 9686 (2015).

18. Yamagata, A. et al. Mechanisms of splicing-dependent trans-synaptic adhesion by PTP $\delta$-IL1RAPL1/IL-1RAcP for synaptic differentiation. Nat. Commun. 6, 6926 (2015).

19. Goto-Ito, S. et al. Structural basis of trans-synaptic interactions between PTP $\delta$ and SALMs for inducing synapse formation. Nat. Commun. 9, 269 (2018).

20. Lin, Z., Liu, J., Ding, H., Xu, F. \& Liu, H. Structural basis of SALM5-induced PTP $\delta$ dimerization for synaptic differentiation. Nat. Commun. 9, 268 (2018).

21. Serra-Pages, C. et al. The LAR transmembrane protein tyrosine phosphatase and a coiled-coil LAR-interacting protein co-localize at focal adhesions. EMBO J. 14, 2827-2838 (1995).

22. Serra-Pages, C., Medley, Q. G., Tang, M., Hart, A. \& Streuli, M. Liprins, a family of LAR transmembrane protein-tyrosine phosphatase-interacting proteins. J. Biol. Chem. 273, 15611-15620 (1998).

23. Weng, Y. L., Liu, N., DiAntonio, A. \& Broihier, H. T. The cytoplasmic adaptor protein Caskin mediates Lar signal transduction during Drosophila motor axon guidance. J. Neurosci. 31, 4421-4433 (2011).

24. Debant, A. et al. The multidomain protein Trio binds the LAR transmembrane tyrosine phosphatase, contains a protein kinase domain, and has separate rac-specific and rho-specific guanine nucleotide exchange factor domains. Proc. Natl Acad. Sci. USA 93, 5466-5471 (1996).

25. Han, K. A. et al. PTP $\sigma$ drives excitatory presynaptic assembly via various extracellular and intracellular mechanisms. J. Neurosci. 38, 6700-6721 (2018).

26. Spangler, S. A. \& Hoogenraad, C. C. Liprin- $\alpha$ proteins: scaffold molecules for synapse maturation. Biochem. Soc. Trans. 35, 1278-1282 (2007).

27. Nachat, R. et al. KazrinE is a desmosome-associated liprin that colocalises with acetylated microtubules. J. Cell Sci. 122, 4035-4041 (2009).

28. Astigarraga, S., Hofmeyer, K., Farajian, R. \& Treisman, J. E. Three Drosophila liprins interact to control synapse formation. J. Neurosci. 30, 15358-15368 (2010).

29. Zhen, M. \& Jin, Y. The liprin protein SYD-2 regulates the differentiation of presynaptic termini in C. elegans. Nature 401, 371-375 (1999).

30. Dai, Y. et al. SYD-2 Liprin- $\alpha$ organizes presynaptic active zone formation through ELKS. Nat. Neurosci. 9, 1479-1487 (2006).

31. Patel, M. R. et al. Hierarchical assembly of presynaptic components in defined C. elegans synapses. Nat. Neurosci. 9, 1488-1498 (2006).

32. Chia, P. H., Patel, M. R., Wagner, O. I., Klopfenstein, D. R. \& Shen, K. Intramolecular regulation of presynaptic scaffold protein SYD-2/liprin- $\alpha$. Mol. Cell Neurosci. 56, 76-84 (2013)

33. Kaufmann, N., DeProto, J., Ranjan, R., Wan, H. \& Van Vactor, D. Drosophila liprin- $\alpha$ and the receptor phosphatase Dlar control synapse morphogenesis. Neuron 34, 27-38 (2002).

34. Choe, K. M., Prakash, S., Bright, A. \& Clandinin, T. R. Liprin- $\alpha$ is required for photoreceptor target selection in Drosophila. Proc. Natl Acad. Sci. USA 103 11601-11606 (2006)

35. Spangler, S. A. et al. Differential expression of liprin- $\alpha$ family proteins in the brain suggests functional diversification. J. Comp. Neurol. 519, 3040-3060 (2011).

36. Zurner, M. \& Mittelstaedt, T., \& tom DieckS. \& Becker A. \& Schoch S. Analyses of the spatiotemporal expression and subcellular localization of liprin-a proteins. J. Comp. Neurol. 519, 3019-3039 (2011).

37. Spangler, S. A. et al. Liprin-a2 promotes the presynaptic recruitment and turnover of RIM1/CASK to facilitate synaptic transmission. J. Cell Biol. 201, 915-928 (2013).

38. Wong, M. Y. et al. Liprin- $\alpha 3$ controls vesicle docking and exocytosis at the active zone of hippocampal synapses. Proc. Natl Acad. Sci. USA 115 , 2234-2239 (2018)

39. Ko, J., Na, M., Kim, S., Lee, J. R. \& Kim, E. Interaction of the ERC family of RIM-binding proteins with the liprin- $\alpha$ family of multidomain proteins. J. Biol. Chem. 278, 42377-42385 (2003).

40. Ko, J. et al. Interaction between liprin- $\alpha$ and GIT1 is required for AMPA receptor targeting. J. Neurosci. 23, 1667-1677 (2003).

41. Shin, H. et al. Association of the kinesin motor KIF1A with the multimodular protein liprin- $\alpha$. J. Biol. Chem. 278, 11393-11401 (2003).

42. Sakamoto, S. et al. Liprin- $\alpha$ controls stress fiber formation by binding to mDia and regulating its membrane localization. J. Cell Sci. 125, 108-120 (2012).

43. Brenig, J. et al. Structural and biochemical basis for the inhibitory effect of liprin- $\alpha 3$ on mouse Diaphanous 1 (mDia1) function. J. Biol. Chem. 290, 14314-14327 (2015)

44. Olsen, O. et al. Neurotransmitter release regulated by a MALS-liprin- $\mathrm{a}$ presynaptic complex. J. Cell Biol. 170, 1127-1134 (2005). 
45. Wei, Z. et al. Liprin-mediated large signaling complex organization revealed by the liprin- $\alpha /$ CASK and liprin- $\alpha /$ liprin- $\beta$ complex structures. Mol. Cell $\mathbf{4 3}$, 586-598 (2011).

46. Almo, S. C. et al. Structural genomics of protein phosphatases. J. Struct. Funct. Genomics 8, 121-140 (2007).

47. Nam, H. J., Poy, F., Krueger, N. X., Saito, H. \& Frederick, C. A. Crystal structure of the tandem phosphatase domains of RPTP LAR. Cell 97, 449-457 (1999).

48. Kamerlin, S. C., Rucker, R. \& Boresch, S. A molecular dynamics study of WPD-loop flexibility in PTP1B. Biochem. Biophys. Res. Commun. 356, 1011-1016 (2007).

49. Barr, A. J. et al. Large-scale structural analysis of the classical human protein tyrosine phosphatome. Cell 136, 352-363 (2009).

50. Buist, A. et al. Restoration of potent protein-tyrosine phosphatase activity into the membrane-distal domain of receptor protein-tyrosine phosphatase $\alpha$. Biochemistry 38, 914-922 (1999).

51. Bomkamp, C. et al. Mechanisms of PTP $\sigma$-mediated presynaptic differentiation. Front. Synaptic Neurosci. 11, 17 (2019).

52. Stafford, R. L. et al. Tandem SAM domain structure of human Caskin1: a presynaptic, self-assembling scaffold for CASK. Structure 19, 1826-1836 (2011).

53. Wyszynski, M. et al. Interaction between GRIP and liprin- $\alpha /$ SYD2 is required for AMPA receptor targeting. Neuron 34, 39-52 (2002).

54. Won, S. Y. et al. LAR-RPTP clustering is modulated by competitive binding between synaptic adhesion partners and heparan sulfate. Front. Mol. Neurosci. 10, 327 (2017).

55. Kim, J. H. et al. High cleavage efficiency of a $2 \mathrm{~A}$ peptide derived from porcine teschovirus-1 in human cell lines, zebrafish and mice. PLoS ONE 6, e18556 (2011).

56. Niwa, H., Yamamura, K. \& Miyazaki, J. Efficient selection for high-expression transfectants with a novel eukaryotic vector. Gene 108, 193-199 (1991).

57. Otwinowski, Z. \& Minor, W. Processing of X-ray diffraction data collected in oscillation mode. Methods Enzymol. 276, 307-326 (1997).

58. Winn, M. D. et al. Overview of the CCP4 suite and current developments. Acta Crystallogr. D Biol. Crystallogr. 67, 235-242 (2011).

59. Vagin, A. \& Teplyakov, A. Molecular replacement with MOLREP. Acta Crystallogr. D Biol. Crystallogr. 66, 22-25 (2010).

60. Emsley, P. \& Cowtan, K. Coot: model-building tools for molecular graphics. Acta Crystallogr D Biol. Crystallogr. 60, 2126-2132 (2004).

61. Adams, P. D. et al. PHENIX: a comprehensive Python-based system for macromolecular structure solution. Acta Crystallogr. D Biol Crystallogr. 66, (213-221 (2010).

62. Krissinel, E. \& Henrick, K. Inference of macromolecular assemblies from crystalline state. J. Mol. Biol. 372, 774-797 (2007).

63. Larkin, M. A. et al. Clustal $\mathrm{W}$ and Clustal X version 2.0. Bioinformatics 23, 2947-2948 (2007).

64. Hashimoto, M. \& Takemoto, T. Electroporation enables the efficient mRNA delivery into the mouse zygotes and facilitates CRISPR/Cas9-based genome editing. Sci. Rep. 5, 11315 (2015)

65. Schneider, C. A., Rasband, W. S. \& Eliceiri, K. W. NIH Image to ImageJ: 25 years of image analysis. Nat. Methods 9, 671-675 (2012).

\section{Acknowledgements}

We thank the beamline staffs of BL41XU of SPring-8 (Hyogo, Japan) for technical help during data collection. This work was supported by grants from JSPS/MEXT KAKENHI (JP 19H03162 to A.Y., JP25293057 to T.Y. and JP18H03983 to S.F.), JST PRESTO to T.Y., and JST CREST (JPMJCR12M5) to S.F.

\section{Author contributions}

M.W. performed crystallography, SPR analysis, and pull-down experiment with assistance from A.Y., S.G.-I., Y.S. and S.F. K.K. performed protein expression experiments for crystallization. A.M. and T.S. constructed the plasmids for cell biological experiments. T.Y. and A.I. performed cell biological experiments. H.I., T.Y., M.S. and H.M. generated knock-in mutant mice. A.Y., S.F. and T.Y. wrote the paper. S.F., A.Y. and T.Y. designed the study. S.F. supervised the study.

\section{Competing interests}

The authors declare no competing interests.

\section{Additional information}

Supplementary information is available for this paper at https://doi.org/10.1038/s41467020-14516-5.

Correspondence and requests for materials should be addressed to T.Y. or S.F.

Peer review information Nature Communications thanks Tommi Kajander and Mingjie Zhang for their contribution to the peer review of this work. Peer reviewer reports are available.

Reprints and permission information is available at http://www.nature.com/reprints

Publisher's note Springer Nature remains neutral with regard to jurisdictional claims in published maps and institutional affiliations.

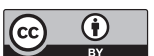

Open Access This article is licensed under a Creative Commons Attribution 4.0 International License, which permits use, sharing, adaptation, distribution and reproduction in any medium or format, as long as you give appropriate credit to the original author(s) and the source, provide a link to the Creative Commons license, and indicate if changes were made. The images or other third party material in this article are included in the article's Creative Commons license, unless indicated otherwise in a credit line to the material. If material is not included in the article's Creative Commons license and your intended use is not permitted by statutory regulation or exceeds the permitted use, you will need to obtain permission directly from the copyright holder. To view a copy of this license, visit http://creativecommons.org/ licenses/by/4.0/.

(C) The Author(s) 2020 